\title{
DISEÑO Y VALIDACIÓN DE LOS CONTENIDOS DE UN INSTRUMENTO PARA EL CONTROL TÉCNICO TÁCTICO DE LA LUCHA OLÍMPICA
}

\section{DESIGN AND VALIDATION OF AN INSTRUMENT CONTENTS TO THE TECHNICAL AND TACTICAL CONTROL IN OLYMPIC WRESTLING}

\author{
Wilfred Stiven Toro Castañeda ${ }^{1}$ y Carlos Alberto Agudelo Velásquez ${ }^{1}$ \\ wilfred.toro@udea.edu.co; carlosa.agudelo@udea.edu.co \\ ${ }^{1}$ Universidad de Antioquia, Medellín, Colombia \\ Envío Original: 2019-06-12 Reenviado: 2020-03-10 Aceptado: 2020-03-19 \\ Publicado: 2020-03-21 \\ Doi: https://doi.org/10.15517/pensarmov.v18i1.37655
}

\begin{abstract}
Resumen
La finalidad de este trabajo fue diseñar y validar los contenidos de una herramienta que permita tener un seguimiento del componente técnico táctico en atletas de lucha olímpica, utilizando los antecedentes de investigaciones relacionadas con esta temática y una validación de un juicio de expertos.
\end{abstract}

La metodología aplicada consistió en aceptar o rechazar contenidos, teniendo en cuenta unos valores de corte $(0,75)$ determinado por el número de evaluadores (8 sujetos que conformaron el panel de expertos en el deporte) y la escala de calificación utilizada en las encuestas (entre 1 y 5), para la valoración de los ítems (11 de la herramienta y 65 de contenidos de esta), a partir de un proceso de validación de contenidos de un instrumento, utilizando el estadístico $\mathrm{V}$ de Aiken.

Los resultados finalmente eliminaron cuatro de 12 ítem del instrumento y siete elementos de los contenidos (cuatro de ellos estaban directamente relacionados con los rechazados en el instrumento), en conclusión, el diseño de una herramienta para observar, controlar y determinar el comportamiento técnico táctico de luchadores olímpicos es de gran importancia, para todos los expertos, pero algunos contenidos se deben eliminar o modificar ya sea por la dificultad en su 
comprensión o porque resultan, según los expertos de poca practicidad. Se infiere la utilidad de una herramienta como la "aprobada" para apoyar el proceso de preparación de los atletas.

Palabras claves: validación, instrumento, control técnico táctico, lucha olímpica, $V$ de Aiken

\begin{abstract}
The purpose of this work was to design and validate the contents of a tool that allows monitoring of the tactical technical component in Olympic wrestling athletes, using the background of research related to this theme and the judgment of experts in said sports modality.

The methodology applied consists of accepting or rejecting contents, taking into account the cutoff values $(0.75)$ determined by the number of evaluators ( 8 subjects Experts in sport) and the rating scale recorded in the surveys (between 1 to 5 ), for the evaluation of the items (11 of the tool and 65 of its contents), based on the validation process of the contents of an instrument, using the Aiken $\mathrm{V}$ statistic.

The paper results finished eliminating four of 12 items of the instrument and seven elements of the contents (four of them were directly related to those rejected in the instrument), in conclusion, the design of a tool for observation, control and determine the tactical technical behavior of Olympic wrestlers is of great importance, for all experts, but some content must be removed or modified either by compression or practicality. The usefulness of a tool such as the "approved" one to support the preparation process of the athletes is inferred.
\end{abstract}

Keywords: validation, instrument, technical - tactical control, olympic wrestling, $\mathrm{V}$ of Aiken

\title{
Introducción
}

La lucha olímpica es uno de los deportes más antiguos, sus orígenes son diversos y lejanos en el tiempo, practicado en la antigua Grecia, este deporte de combate consiste en dominar al oponente por medio de acciones de agarres, lanzamientos y derribos (López-Gullón y MartínezAbellán, 2011). Lo que permite caracterizar a esta disciplina deportiva en una categoría de "cuerpo a cuerpo" según Henares (2000), lo que significa, que no hay golpes provocados por patadas o puños y los combates se ganan por puntuación o por "plancha" (fall en inglés) que consiste en poner al rival de espaldas contra la colchoneta donde se compite (United World Wrestling, 2017). 
Tornello, Capranica, Chiodo, Minganti y Tessitore (2013) afirman que es necesario determinar el comportamiento en el contexto de combate para apoyar y fundamentar mejor el diseño de programas de entrenamiento técnico-táctico en lucha olímpica de forma que estén vinculadas las características fisiológicas y psicológicas de los deportistas, con su real desempeño técnicotáctico, a pesar de este hallazgo son pocos los trabajos de investigación relacionados con este tema, y todos los que se lograron encontrar serán mencionados como respaldo para el objetivo de este estudio: diseño de una herramienta para analizar atletas de lucha y sus respectivos contenidos.

Un estudio comparativo del rendimiento en competencia de luchadores olímpicos entre los Juegos Olímpicos de Beijíng 2008 y Londres 2012, mostró una disminución en el número de acciones ejecutadas y efectivas, es decir, se observó un menor rendimiento, al de las olimpiadas anteriores, se concluyó que fue debido al cambio del reglamento (Tropin, 2013).

Sin embargo, el comportamiento en competencia es una evaluación pedagógica que permite obtener un panorama mucho más amplio respecto a las demandas técnico-tácticas y fisiológicas de los atletas (Bridge, Jones y Drust, 2011), esto nos dice que, dicha "evaluación pedagógica" tendría que ser adaptativa y no podría verse afectada por algo tan común (en la lucha olímpica) como el cambio del reglamento.

López-González (2010) logró diseñar una herramienta que permite caracterizar las combinaciones de acciones técnico-tácticas de pie (CCT), este instrumento fue realizado a través del análisis de videos del campeonato mundial femenino de 2009, lo que conllevó a desarrollar algunas categorías y su evaluación pedagógica, tuvo en cuenta variables como el riesgo de las acciones, la diversidad, el tipo de acción técnica, la efectividad de la acción y preparación. Su propuesta de clasificación se dividió en tres fases: 1. Tipo de preparación, que comprende cuáles serán las estrategias para generar oportunidades. 2. Técnica o ejecución (grupo técnico) donde se clasifican las CCT según el gesto técnico a partir de un control sobre el adversario (agarre). 3. Final (Efectividad), donde se evalúa la obtención de puntos a partir de un gesto técnico efectivo.

El estudio concluyó que la variabilidad con las que se ejecutan las acciones técnico - tácticas permite tener mayor éxito y un mejor rendimiento en competencia, a diferencia de un estado ofensivo enfocado en acciones específicas (ataque a una y dos piernas) (López-González, 2010; López-González, 2011). 
En busca de mejorar la herramienta diseñada en 2010, López-González, Alonso, Bárcenas y Alonso (2012) adicionan elementos como el coeficiente pie vs piso propuesto por Podlivaev (2010), que establece relaciones entre las acciones de pie y acciones de piso, lo que da lugar a que aparezcan elementos como proactividad táctica en posición de pie y la distancia media efectiva de pie, que permiten reconocer y cuantificar la variabilidad de las acciones técnicas de los campeones.

Esta herramienta fue modificada y analizada nuevamente, con la intención de evaluar la confiabilidad de esta y su practicidad en unión con un análisis del tiempo de ejecución de las acciones técnicas (López-González y Miarka, 2013).

Finalmente, esta sería la única herramienta utilizada y estudiada para cuantificar las CCT, hasta el momento. No obstante, en el estudio de López-González y Miarka (2013), no se realizó validación de contenidos, por ejemplo a través de un panel de expertos, aspecto que si se logró en otras publicaciones en el área deportiva, pero en disciplinas diferentes, como lo hizo Conejero, Claver, Fernández-Echavarría, González-Silva y Moreno (2017) para valorar la toma de decisiones en las acciones de recepción de voleibol, o Santos e Ibáñez (2016) para valorar el desempeño de los árbitros de baloncesto, ambos a través del coeficiente $V$ de Aiken diseñado por Aiken (1985).

Esta es la razón por la que este estudio retoma el tema, construyendo un proceso de validez de los contenidos de una herramienta, pero a su vez tratando de proponer algunos contenidos que permitan incluir de forma general las dos modalidades: Libre y Greco para el control técnicotáctico basada en los antecedentes descritos y apoyada en recomendaciones de expertos.

\section{Material y Método}

Diseño

El diseño de este estudio encuadra en el campo científico de las ciencias del entrenamiento (Borms, 2008), específicamente en el área del análisis del rendimiento deportivo, en este caso, el análisis de la técnica, relacionada con la efectividad y el perfil de rendimiento (O'Donoghue, 2010). Es un diseño instrumental y ex post facto (Montero y León, 2007). La finalidad del estudio es la validación de los contenidos de un instrumento para el control de las acciones técnicotácticas en combates de lucha olímpica, a través del criterio de un panel de expertos. 


\section{Participantes}

Se recurre a una muestra diversa, compuesta por entrenadores, atletas de alto rendimiento y jueces nacionales e internacionales, cuyos criterios de selección se especifican la tabla 1. La población fue elegida a conveniencia, y se aplicó un censo poblacional, para el recuento de los participantes en el proceso de validación, ya que el número de participantes determina, los valores de corte, para aceptación o rechazo, de los contenidos de la herramienta; obtener una muestra mayor a cinco evaluadores, fue uno de los ideales del estudio, de acuerdo con Lynn (1986), para que los resultados puedan tener una solidez estadística.

Tabla 1.

Criterios de inclusión de expertos.

\section{Criterios de inclusión para Entrenadores}

C1. Experiencia mayor a 5 años como entrenador

C2. Logros deportivos recientes (últimos dos años), mínimo nivel nacional (donde haya ganado primer puesto por modalidades o primer puesto en general)

C3. Formación Académica Tecnológica o Profesional. (Aplica para los que aún son estudiantes)

\section{Criterios de inclusión para Atletas}

C1. Participación en Competencias Internacionales del ciclo olímpico (Panamericanos o superiores), pertenecientes a la Selección Nacional, senior

C2. Experiencia deportiva superior a 7 años, (Para facilitar la comprensión de temas mencionados en la validación, que van más allá de lo operacional o técnico).

\section{Criterios de inclusión para Jueces}

C1. Poseer licencia nacional o superior, aprobado por la UWW (United World Wrestling)

C2. Experiencia mayor a 5 años.

Fuente: elaboración propia

\section{Procedimiento}

Se entregó una encuesta a los expertos donde estos debían valorar cada ítem del instrumento y sus contenidos, en una escala cualitativa de 5 valores de importancia, que iban de irrelevante (1), poco importante (2), importante (3), muy importante (4) o imprescindible (5), acompañado de una casilla de observaciones donde estos podrían dar sugerencias u opiniones para mejorar la 
herramienta, como en estudios anteriores (López-González y Miarka, 2013; López-González, 2010; López-González, 2011; Lopez, Alonso, Bárcenas, y Alonso, 2012; Podlivaev, 2010), e incluyendo elementos técnicos de pie y piso propios de la modalidad Greco, así como: el área, la orientación (lateralidad predominante de las acciones técnicas), la victoria por plancha o fall y la calidad de las acciones técnicas con su efectividad ofensiva o defensiva diferenciada.

Análisis de los datos

Se usó el estadístico $V$ de Aiken para determinar la aceptación o rechazo de los ítems, en una hoja de cálculo del programa Excel de Microsoft Office, y el valor de corte se estableció teniendo en cuenta la escala de calificación (1 a 5) y el número de jueces que hicieron parte del panel de expertos: ocho (8 participantes).

\section{Resultados}

Para la validación de los contenidos de la herramienta de Lucha Olímpica se contó con la participación de ocho expertos (Tabla 2), quienes valoraron la relevancia de los ítems, los resultados de su valoración se pueden observar en la Tabla 3 . La muestra de expertos fue de tres jueces internacionales, tres atletas de alto rendimiento y dos entrenadores de selección departamental y nacional, los cuales cumplen con la totalidad de los criterios de selección establecidos.

Los tres expertos considerados del grupo de entrenadores; al igual que los dos atletas y los dos jueces cumplieron con todos los criterios descritos en la Tabla 1. 
Tabla 2.

Resultado del cálculo de la validez de contenidos con $V$ de Aiken

\begin{tabular}{clcl}
\hline No. de Ítem & Denominación del Ítem & V Obtenida & Resultado \\
\hline 1 & Efectividad Ofensiva & 0,89 & Aceptado \\
2 & Efectividad Defensiva & 0,82 & Aceptado \\
3 & Efectivas/minuto & 0,93 & Aceptado \\
4 & Productividad & 0,79 & Aceptado \\
5 & Coeficiente Pie/Piso & 0,96 & Aceptado \\
6 & Productividad Táctica de & 0,93 & Aceptado \\
7 & Pie & 0,75 & Aceptado \\
8 & Distancia media efectiva & 0,71 & Rechazado \\
9 & Riesgo Promedio & 0,71 & Rechazado \\
10 & Acciones en zona Central & 0,61 & Rechazado \\
11 & Acciones en zona de & 0,61 & Rechazado \\
\hline
\end{tabular}

Fuente: elaboración propia

En la tabla 2 se observan los resultados de la valoración de los ítems a valorar por parte de los expertos para Lucha Olímpica, se realizaron mediante el Coeficiente $V$ de Aiken y el resultado obtenido para ser aceptado tiene que ser: $V=0.75$ con $p=0.030$, de acuerdo, con el número de expertos y la escala de valoración de los ítems y contenidos. (Aiken, 1985).

Respecto a los contenidos de la herramienta se eliminaron dos elementos técnicos: "agarre doble de muñecas" y cinco elementos tácticos referentes a la "orientación de las acciones" y "el contacto inicial" como se muestra en las tablas $\underline{3}$ y $\underline{4}$. 
Tabla 3.

Ítems de los contenidos pertenecientes a los elementos técnicos.

\begin{tabular}{|c|c|c|c|c|c|}
\hline Ítem & Dimensión & Denominación del Ítem & $\mathbf{m}$ & m d & m téc \\
\hline 1 & & Lanzamiento de Brazo & 0,75 & & \\
\hline 2 & & Levantamiento de bombero & 0,78 & & \\
\hline 3 & & Proyección de cadera & 0,81 & & \\
\hline 4 & Proyecciones & Suplex & 0,81 & 0,8 & \\
\hline 5 & & Uchi mata & 0,84 & & \\
\hline 6 & & Levantamiento entrepierna o cintura ("turca") & 0,84 & & \\
\hline 7 & & Tacles a tobillo & 0,81 & & \\
\hline 8 & & Tacles a dos piernas & 0,88 & & \\
\hline 9 & & Tacles a una pierna & 0,78 & & \\
\hline 10 & Derribes & Barridos de pie & 0,78 & 0,8 & \\
\hline 11 & & Enganches de pierna & 0,81 & & \\
\hline 12 & & Tomas de cintura & 0,81 & & \\
\hline 13 & & Media nelson & 0,78 & & \\
\hline 14 & & Cuarto de nelson & 0,75 & & \\
\hline 15 & & Viraje por pecho & 0,84 & & \\
\hline 16 & & Viraje por cintura & 0,81 & & \\
\hline 17 & Suelo o piso & Viraje con cruce de piernas & 0,84 & 0,8 & \\
\hline 18 & & Viraje de cabeza y brazo & 0,81 & & \\
\hline 19 & & Viraje con cruce de rostro & 0,75 & & 0,8 \\
\hline 20 & & Levantamiento de entrepierna ("turca") & 0,84 & & \\
\hline 21 & & Combinaciones & 0,84 & & \\
\hline 22 & & Agarre doble de muñecas & 0,72 & & \\
\hline 23 & & Agarre de muñeca & 0,78 & & \\
\hline 24 & & 2 a 1 ruso & 0,81 & & \\
\hline 25 & & Muñeca a brazo (1:2) & 0,78 & & \\
\hline 26 & & Subaxilar simple & 0,84 & & \\
\hline 27 & & Subaxilar doble & 0,78 & & \\
\hline 28 & & Subaxilar y muñeca & 0,75 & & \\
\hline 29 & Controles & Agarre lateral de cintura & 0,78 & & \\
\hline 30 & Bloqueos & Agarre doble de cintura & 0,75 & 0,8 & \\
\hline 31 & & Agarre doble a muñeca (una sola) & 0,81 & & \\
\hline 32 & & Evasión con ambas piernas & 0,88 & & \\
\hline 33 & & Evasión con una pierna & 0,88 & & \\
\hline 34 & & Agarre de tobillo & 0,84 & & \\
\hline 35 & & Rotacional & 0,88 & & \\
\hline 36 & & Extensión de brazos & 0,88 & & \\
\hline 37 & & Ninguno & 0,59 & & \\
\hline
\end{tabular}

Nota: ${ }^{*} \mathrm{~m}$ : media del valor $\mathrm{V}$ de Aiken; $\mathrm{m}$ d: media de la dimensión; $\mathrm{m}$ téc: media de los ítems técnicos. Fuente: elaboración propia

Se evidencia que en lo que corresponde al contenido técnico de controles y bloqueos, los expertos concluyen que el "agarre doble de muñecas" y el no lograr "ningún" control o bloqueo (dentro de un contexto de lucha defensiva) son elementos que no tienen tanta importancia al 
momento de analizar un combate, lo que respecta al contenido táctico, se rechazó el medio circunstancial de "oportunidad" y sobre la orientación de las acciones, se eliminaron cuatro de los seis totales lo que se puede interpretar en general como un rechazo a esta dimensión. Como se observa en las Tablas $\underline{2}$ y $\underline{3}$.

Tabla 4.

Ítem de los contenidos pertenecientes a los elementos tácticos.

\begin{tabular}{|c|c|c|c|c|c|}
\hline Ítems & Dimensión & Denominación del Ítem & $\mathbf{m}$ & m d & m tác \\
\hline 1 & & Movimiento & 0,81 & \multirow{8}{*}{0,8} & \\
\hline 2 & & Finta & 0,84 & & \\
\hline 3 & & Empujes y Tracciones & 0,81 & & \\
\hline 4 & Medios & Desvió & 0,81 & & \\
\hline 5 & \multirow[t]{5}{*}{ tácticos } & Pasividad & 0,84 & & \\
\hline 6 & & Castigo & 0,88 & & \\
\hline 7 & & Oportunidad & 0,72 & & \\
\hline 8 & & Ataque Directo & 0,84 & & \\
\hline 9 & & Central & 0,84 & \multirow{3}{*}{0,8} & \\
\hline 10 & \multirow[t]{2}{*}{ Espacio } & Zona de pasividad & 0,81 & & \\
\hline 11 & & Zona de protección & 0,78 & & \\
\hline 12 & \multirow{2}{*}{ Puntuación } & Puntos de rival & 0,88 & \multirow{2}{*}{0,9} & \\
\hline 13 & & Puntos obtenidos & 0,88 & & \\
\hline 14 & \multirow{3}{*}{ Posición } & Pie & 0,84 & \multirow{3}{*}{0,8} & \\
\hline 15 & & Piso & 0,84 & & 0,8 \\
\hline 16 & & Bajo & 0,88 & & \\
\hline 17 & \multirow{3}{*}{ Riesgo } & Medio & 0,84 & \multirow{3}{*}{0,9} & \\
\hline 18 & & Alto & 0,94 & & \\
\hline 19 & & Muy alto & 0,88 & & \\
\hline 20 & \multirow{3}{*}{$\begin{array}{l}\text { Contacto } \\
\text { inicial }\end{array}$} & Sin Contacto & 0,81 & \multirow{3}{*}{0,8} & \\
\hline 21 & & Rápida & 0,75 & & \\
\hline 22 & & De poder & 0,91 & & \\
\hline 23 & \multirow{6}{*}{ Orientación } & Derecha & 0,72 & \multirow{6}{*}{0,7} & \\
\hline 24 & & Izquierda & 0,63 & & \\
\hline 25 & & Interno & 0,63 & & \\
\hline 26 & & Externo & 0,63 & & \\
\hline 27 & & Anterior & 0,78 & & \\
\hline 28 & & Posterior & 0,78 & & \\
\hline
\end{tabular}

Nota: ${ }^{*} \mathrm{~m}$ : media del valor $\mathrm{V}$ de Aiken; $\mathrm{m}$ d: media de la dimensión; $\mathrm{m}$ tác: media de los ítems tácticos. Fuente: elaboración propia 


\section{Discusión}

Los ítems evaluados son el resultado de los aportes de las investigaciones, las sugerencias de algunos expertos y la edición de algunas variables estudiadas, como se observó no todos los elementos de la herramienta de análisis fueron aceptados, como en Santos e Ibáñez (2016) ya que se eliminaron los ítems de riesgo promedio, calidad de las acciones y acciones realizadas en zona central y en zona de pasividad, los demás indicadores son aceptados por el grupo de expertos.

Conejero et al. (2017) indican que la mayoría de autores recomiendan un mínimo de 10 expertos para que la validez de los contenidos de una herramienta tenga más potencia y solidez, no obstante, de acuerdo a lo propuesto por Aiken (1985) se pueden realizar estas validaciones hasta con tres expertos, Lynn (1986) establecen que un mínimo de tres evaluadores es suficiente para realizar una validación de contenidos y cinco es un número aceptable, el número de expertos obtenido para este estudio fue de ocho, por lo que se afirma que al tamaño de la muestra de expertos fue adecuada y suficiente.

Se establecieron unos valores de corte de $\mathrm{V}$ para de 0,75 donde los ítems con valores inferiores a este se eliminan y aquellos que sean superiores se aceptan, en diferentes trabajos donde se utilizaron estas metodologías el punto de corte varía desde 0,66 a 0,7 en razón del número de ítems y el número de jueces evaluadores. Para este caso se definió este límite de acuerdo con el diseño de los estudios donde se tuvo en cuenta el número de evaluadores y escala de calificación (Aravamudhan y Krishnaveni, 2015; Napitupulu, Syafrullah, Rahim, Amar y Sucahyo, 2018; Yang, 2011).

En investigaciones como las mencionadas se terminó rechazando entre uno y 11 ítems, en el caso de Santos y lbañez (2016), se eliminaron cuatro de 11 (36,3\%), lo que significa que más de una tercera parte de los contenidos no cumplía con el criterio de los expertos, en este estudio se eliminaron sólo siete ítems de 65 , es decir el $11 \%$, esto muestra una buena aceptación de contenidos por parte de los expertos, el contenido: "orientación del gesto técnico" fue rechazado por la mayoría de atletas, quienes aseguran que no tiene importancia ya que "un rival zurdo o distro deberá aprender a ejecutar las técnicas por ambos lados", y el otro que contó con poca popularidad fue: en la dimensión de controles y bloqueos la categoría de: "ninguno" porque aun si es fallido "toda acción técnica siempre debe tener oposición del rival, aun si esta es poco efectiva" 
En García-Martin et al. (2016) la fuerte eliminación de contenidos del instrumento puede ser atribuible a la falta de información previa entregada a los expertos sobre el instrumento a validar, esta conclusión puede ser también válida para este estudio ya que se trata de un tema exploratorio, unido a que actualmente no se cuenta con una herramienta clara y precisa del rendimiento técnico-táctico de los atletas de lucha que permita a los expertos estar habituados con un instrumento de estas características, lo que lo hace especialmente importante, ya que puede aportar al seguimiento individual que es tan importante en los nuevos modelos de periodización (Agudelo, 2012; Costa, 2013).

Los ítems evaluados son el resultado de los aportes de las investigaciones, las sugerencias de algunos expertos y la edición de algunas variables estudiadas es dichos trabajos de investigación, como se observa no todos los elementos de la herramienta de análisis fueron aceptados, eliminando algunos ítems, como el riesgo promedio, calidad de las acciones y acciones realizadas en zona central y en zona de pasividad, todos los demás indicadores se aceptaron como importantes para la herramienta por el grupo de expertos, algunos de los ítem eliminados tuvieron sugerencias de asociación entre ellos, como establecer un coeficiente entre acciones en zona de pasividad y zona central y mejorar algunos conceptos, para una mejor comprensión.

A pesar de que los criterios de inclusión se cumplieron con el total de participantes, los jueces manifestaron dificultad para comprender la encuesta, de que se trataba y algunos conceptos que parece ser, podrían representar ambigüedad o mala interpretación. Dichas modificaciones tendrían más relevancia en la medida que se incluyan nuevos evaluadores y un grupo de trabajo más grande en el proceso del diseño y validación de esta herramienta, puede ser importante para próximos estudios en el tema aumentar el número de expertos y ubicarlos de varias nacionalidades.

\section{Conclusiones}

Con este estudio se concluye que el diseño de una herramienta para observar, controlar y determinar el comportamiento técnico táctico de luchadores olímpicos es reconocida como importante, por los entrenadores atletas y jueces. El panel de expertos compuesto por estos tres estamentos es una fortaleza, ya que incluye diferentes perspectivas y permitió llegar a la definición de que algunos aspectos que se tuvieron presentes en la propuesta inicial, no 
resultaron tan relevantes y por tanto deben ser descartados o redactados y relacionados de una manera diferente. Se puede inferir que poseer una herramienta como la que se propone va a ser muy útil para adelantar una preparación más eficiente, ya que permitirá estar observando qué aciertos y aspectos a mejorar debe ir teniendo el luchador en su proceso individual de preparación.

\section{Referencias}

Agudelo, C.A. (2012). Planificación del entrenamiento deportivo por Modelamiento. Kinesis: Armenia. Recuperado de https://editorial-kinesis.com/producto/planificacion-delentrenamiento-deportivo-por-modelamiento/

Aiken, L. R. (1985). Three coefficients for analyzing the reliability and validity of ratings. Educational and Psychological Measurement, 45(1), 131-142. doi: https://doi.org/10.1177/0013164485451012

Aravamudhan, N. R., y Krishnaveni, R. (2015). Establishing and reporting content Validity Evidence of New Training and Development Capacity Building Scale (Tdcbs). Management, 20(1), 131-158. Recuperado de https://www.semanticscholar.org/paper/Establishing-and-Reporting-Content-Validity-ofNew-Aravamudhan-Krishnaveni/d7e2be222d4fb1f4816472d402967ba404b6ac36

Borms, J. (2008). Directory of Sport Science. Berlin: Human Kinetics. Recuperado de https://bit.ly/2UeVgIQ

Bridge, C. A., Jones, M. A., y Drust, B. (2011). The activity profile in international taekwondo competition is modulated by weight category. International journal of sports physiology and performance, 6(3), 344-357. doi: https://doi.org/10.1123/ijspp.6.3.344

Conejero, M., Claver, F., Fernández-Echavarría, C., González-Silva, J., y Moreno, P. (2017). Diseño y validación de un instrumento de observación para valorar la toma de decisiones en la acción de recepción en voleibol. Cultura, ciencia y deporte, 12(34), 67-75. doi: http://dx.doi.org/10.12800/ccd.v12i34.833

Costa, I.A. (2013). Los Modelos de planificación del entrenamiento deportivo del siglo XX. Revista Electrónica de Ciencias Aplicadas al Deporte, 6(22), 1-8. Recuperado de https://core.ac.uk/download/pdf/49224383.pdf

García-Martin, A., Antúnez, A., y lbáñez, S. J. (2016). Analysis of expert players'training process: validation of tools. Revista Internacional de Medicina y Ciencias de la Actividad Física y del Deporte, 16(61), 157-181. doi: http://dx.doi.org/10.15366/rimcafd2016.61.012

Henares, J. (2000). Análisis de la estructura y dinámica de los deportes. En J. Hernández. La iniciación a los deportes desde su estructura dinámica (pp. 21-73). Barcelona: INDE.

López-González, D. E. (2010). Caracterización de las combinaciones técnico/tácticas en posición de pie realizadas con efectividad en el campeonato del mundo senior de lucha femenil 2009. Revista de Ciencias Del Ejercicio, 6, 29-31. Recuperado de https://bit.ly/2J2S1iy 
López-González, D. E. (2011). Factores determinantes de la frecuencia de combinaciones técnico/tácticas efectivas en la lucha de pie durante el campeonato del mundo senior femenil 2009. Revista de Ciencias Del Deporte, 7, 63-74. Recuperado de http://www.ebalonmano.com/ojs/index.php/revista/article/view/76/69

López, D., Alonso, A., Bárcenas, M., y Alonso, S. (2012). Evaluación cuantitativa del rendimiento técnico-táctico en la lucha libre olímpica. Revista mexicana de investigación en cultura física $y$ deporte, 4(5), 143-159. Recuperado de http://revista.ened.edu.mx/index.php/revistaconade/article/view/88

López-González, D. E., y Miarka, B. (2013). Reliability of A New Time-Motion Analysis Model Based on Technical-Tactical Interactions for Wrestling Competition. International Journal of Wrestling Science, 3(1), 21-34. doi: https://doi.org/10.1080/21615667.2013.10878967

López-Gullón, J. M., y Martínez-Abellán, A. (2011). Evolución del reglamento oficial de luchas olímpicas y sus consecuencias sobre las capacidades condicionales y habilidades técnicotácticas. e-balonmano.com: Revista de Ciencias Del Deporte, 7, 39-44. Recuperado de https://dialnet.unirioja.es/descarga/articulo/3639288.pdf

Lynn, M. (1986). Determination and quantification of content validity. Nursing Research, 35(6), 382-385. doi: http://dx.doi.org/10.1097/00006199-198611000-00017

Montero, I. G., y León, O. (2007). A guide for naming research studies in Psychology. International Journal of Clinical and Health Psychology, 7(3), 847-862. Recuperado de: http://www.aepc.es/ijchp/GNEIP07 es.pdf

Napitupulu, D., Syafrullah, M., Rahim, R., Amar, A. y Sucahyo, Y. G. (2018). Content validity of critical success factors for e-Government implementation in Indonesia. Ponencia presentada en IOP Conference Series: Materials Science and Engineering. Recuperado de https://iopscience.iop.org/article/10.1088/1757-899X/352/1/012058/meta

O'Donoghue, P. (2010). Métodos de investigación para el análisis del rendimiento deportivo. Editorial Routledge. Recuperado de https://books.google.com.co/books?id=9Kd5AgAAQBAJ

Podlivaev, B. A. (2010). The concept of top-level wrestlers training. In: modern problems of high quality training in wrestling (pp. 63-78). Moscow: Russian Wrestling Federation.

Santos, D., y lbáñez, S. J. (2016). Diseño y validación de un instrumento de observación para la valoración de un árbitro de baloncesto (IOVAB). Sport-TK Revista Euroamericana de Ciencias del Deporte, 5(2), 15-26. doi: https://doi.org/10.6018/264601

Tornello, F., Capranica, L., Chiodo, S., Minganti, C., y Tessitore, A. (2013). Time-motion analysis of youth Olympic Taekwondo combats. The Journal of Strength \& Conditioning Research, 27(1), 223-228. doi: https://doi.org/10.1519/JSC.0b013e3182541edd

Tropin, G. (2013). Comparative analysis of technical and tactical preparedness greco-roman style wrestler at the Olympic Games-2008 and the Olympic Games-2012. Journal Contribution. doi: https://doi.org/10.6084/m9.figshare.663629

United World Wrestling. (2017). Reglamento Internacional de Lucha. Recuperado de https://unitedworldwrestling.org/sites/default/files/media/document/wrestling rules esp 0 .pdf 
Yang, W.C. (2011). Applying Content Validity Coefficient and Homogeneity Reliability Coefficient to Investigate the Experiential Marketing Scale for Leisure Farms. ArXiv, 1-8. Recuperado de https://www.semanticscholar.org/paper/Applying-Content-Validity-Coefficient-and-tothe-Yang/f277e3488a7b79395cfd3cd32445f6d94e44ec18 


\section{Anexos}

\begin{tabular}{|c|c|c|c|c|c|c|c|c|c|c|c|c|c|c|c|}
\hline \multicolumn{2}{|l|}{ Atleta } & \multicolumn{4}{|l|}{ SUJETO 1} & & \multicolumn{2}{|l|}{ Modalidad } & GR & Categoria & \multirow[t]{2}{*}{60} & \multicolumn{4}{|c|}{ Pais } \\
\hline \multicolumn{2}{|c|}{ TIEMPO } & \multicolumn{9}{|c|}{ DATOS DEL ATLETA (LUCHADOR) } & & \multicolumn{4}{|c|}{ DATOS DEL RIVAL } \\
\hline Acumulado & $\begin{array}{c}\text { Entre } \\
\text { Acciones }\end{array}$ & $\begin{array}{l}\text { Tipo de } \\
\text { Acción }\end{array}$ & Técnica & Elemento & $\begin{array}{c}\text { Contacto } \\
\text { Inicial }\end{array}$ & Orientación & $\begin{array}{c}\text { Estado de la } \\
\text { acción }\end{array}$ & Riego & $\begin{array}{l}\text { Medios } \\
\text { tacticos }\end{array}$ & $\begin{array}{c}\text { Puntos } \\
\text { obtenidos }\end{array}$ & $\begin{array}{c}\text { Lugar de } \\
\text { acción }\end{array}$ & $\begin{array}{l}\text { Técnica del } \\
\text { Oponente }\end{array}$ & $\begin{array}{l}\text { Elemento } \\
\text { OPONENTE }\end{array}$ & $\begin{array}{l}\text { Control / } \\
\text { Bloqueo }\end{array}$ & $\begin{array}{c}\text { Puntos en } \\
\text { contra }\end{array}$ \\
\hline 8 & 8 & & & & & & & & & & & & & & \\
\hline 122 & 114 & Ofensiva & Proyecciones & $\begin{array}{l}\text { Proyeccion de } \\
\text { cadera }\end{array}$ & De poder & Izquierda & $\begin{array}{l}\text { Incompleta } \\
\text { sin } \\
\text { marcación }\end{array}$ & Alto & $\begin{array}{l}\text { Ataque } \\
\text { Directo }\end{array}$ & 0 Central & Pie & Controles & $\begin{array}{l}\text { Agarre doble de } \\
\text { cintura }\end{array}$ & Ninguno & 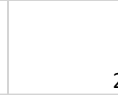 \\
\hline 126 & 4 & \begin{tabular}{|l|} 
Contra \\
ataque
\end{tabular} & Controles & $\begin{array}{l}\text { Agarre doble de } \\
\text { cintura }\end{array}$ & De poder & Anterior & Completa & Alto & $\begin{array}{l}\text { Ataque } \\
\text { Directo }\end{array}$ & $\begin{array}{c}\text { Zona de } \\
2 \text { pasividad }\end{array}$ & Pie & Controles & Ninguno & & \\
\hline 136 & 10 & Ofensiva & Suelo & Viraje por cintura & De poder & Posterior & $\begin{array}{l}\text { Incompleta } \\
\text { sin } \\
\text { marcación }\end{array}$ & Muy alto & $\begin{array}{l}\text { Ataque } \\
\text { Directo }\end{array}$ & 0 Central & Piso & Controles & $\begin{array}{l}\text { Extension de } \\
\text { brazos }\end{array}$ & & \\
\hline 140 & 4 & $\begin{array}{l}\text { Contra } \\
\text { ataque }\end{array}$ & Proyecciones & Suplex & De poder & Anterior & Completa & Muy alto & Desvio & 4 Central & Pie & Controles & Rotacional & & c \\
\hline 203 & 63 & Defensiva & Controles & Rotacional & De poder & Anterior & Completa & Alto & Movimiento & 0 Central & Pie & Proyecciones & Suplex & Rotacional & 2 \\
\hline 251 & 48 & Defensiva & Controles & $\begin{array}{l}\text { Subaxilary } \\
\text { muñeca }\end{array}$ & De poder & Anterior & $\begin{array}{l}\text { Incompleta } \\
\text { sin } \\
\text { marcación }\end{array}$ & Medio & Desvio & 0 Central & Pie & Derribos & Toma de cintura & $\begin{array}{l}\text { Subaxilary } \\
\text { muñeca }\end{array}$ & c \\
\hline 270 & 19 & & & & & & & & & 0 & & Proyecciones & Suplex & Ninguno & ( \\
\hline 321 & 51 & $\begin{array}{l}\text { Contra } \\
\text { ataque }\end{array}$ & Controles & Subaxilar doble & De poder & Anterior & Completa & Medio & Movimiento & $\begin{array}{l}\text { Zona de } \\
2 \text { pasividad }\end{array}$ & Pie & Derribos & Toma de cintura & & \\
\hline 333 & 12 & Ofensiva & Proyecciones & Suplex & De poder & Posterior & Completa & Muy alto & $\begin{array}{l}\text { Ataque } \\
\text { Directo }\end{array}$ & $\begin{array}{c}\text { Zona de } \\
2 \text { pasividad } \\
\end{array}$ & Piso & Controles & Rotacional & & c \\
\hline segundos & minutos & & & & & 3 & & & 3 & 4 total ptos & 6 & & & & total ptos \\
\hline 333 & 5,6 & & & & & & & & & $\begin{array}{ll}4 & 10\end{array}$ & 2 & 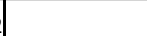 & & & 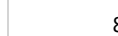 \\
\hline
\end{tabular}

Figura 1. Prototipo inicial de la herramienta, previo a su proceso de validación de contenidos. Fuente: elaboración propia

Se observan todos los componentes descritos en el estudio, ejecutados por el atleta y el rival durante un combate y su respectiva descripción y análisis. Se tiene en cuenta el momento del combate (tiempo) en el que lo ejecutó la acción técnica, en qué dirección, con que agarre, su calidad de ejecución, el medio utilizado para lograrlo, los puntos obtenidos y el área donde se realizó, paralelamente se observa el comportamiento del oponente que permitirá evaluar los aspectos defensivos del atleta y caracterizar al rival. 


\begin{tabular}{|c|c|c|c|c|c|c|c|c|c|c|c|c|}
\hline \multicolumn{2}{|l|}{ Atleta } & \multicolumn{4}{|l|}{ SUJETO 1} & GR & Categoria & 60 & & \multicolumn{3}{|l|}{ Pais } \\
\hline \multicolumn{2}{|c|}{ TIEMPO } & \multicolumn{7}{|c|}{ DATOS DEL ATLETA (LUCHADOR) } & \multicolumn{4}{|c|}{ DATOS DEL RIVAL } \\
\hline Acumulado & $\begin{array}{c}\text { Entre } \\
\text { Acciones }\end{array}$ & $\begin{array}{l}\text { Tipo de } \\
\text { Acción }\end{array}$ & Técnica & Elemento & $\begin{array}{c}\text { Contacto } \\
\text { Inicial }\end{array}$ & $\begin{array}{l}\text { Medios } \\
\text { tacticos }\end{array}$ & $\begin{array}{c}\text { Puntos } \\
\text { obtenidos }\end{array}$ & $\begin{array}{l}\text { Lugar de } \\
\text { acción }\end{array}$ & $\begin{array}{l}\text { Técnica del } \\
\text { Oponente }\end{array}$ & $\begin{array}{l}\text { Elemento } \\
\text { OPONENTE }\end{array}$ & $\begin{array}{l}\text { Control / } \\
\text { Bloqueo }\end{array}$ & $\begin{array}{c}\text { Puntos en } \\
\text { contra }\end{array}$ \\
\hline 8 & 8 & & & & & & & & & & & \\
\hline 122 & 114 & Ofensiva & Proyecciones & $\begin{array}{l}\text { Proyeccion de } \\
\text { cadera }\end{array}$ & De poder & $\begin{array}{l}\text { Ataque } \\
\text { Directo }\end{array}$ & \multicolumn{2}{|c|}{0 Pie } & Controles & $\begin{array}{l}\text { Agarre doble de } \\
\text { cintura }\end{array}$ & Ninguno & 2 \\
\hline 126 & 4 & $\begin{array}{l}\text { Contra } \\
\text { ataque }\end{array}$ & Controles & $\begin{array}{l}\text { Agarre doble de } \\
\text { cintura }\end{array}$ & De poder & $\begin{array}{l}\text { Ataque } \\
\text { Directo }\end{array}$ & \multicolumn{2}{|c|}{2 Pie } & Controles & Ninguno & & 0 \\
\hline 136 & 10 & Ofensiva & Suelo & Viraje por cintura & De poder & $\begin{array}{l}\text { Ataque } \\
\text { Directo }\end{array}$ & \multicolumn{2}{|c|}{0 Piso } & Controles & $\begin{array}{l}\text { Extension de } \\
\text { brazos }\end{array}$ & & 0 \\
\hline 140 & 4 & $\begin{array}{l}\text { Contra } \\
\text { ataque }\end{array}$ & Proyecciones & Suplex & De poder & Desvio & \multicolumn{2}{|c|}{4 Pie } & Controles & Rotacional & & 0 \\
\hline 203 & 63 & Defensiva & Controles & Rotacional & De poder & Movimiento & \multicolumn{2}{|c|}{0 Pie } & Proyecciones & Suplex & Rotacional & 2 \\
\hline 251 & 48 & Defensiva & Controles & $\begin{array}{l}\text { Subaxilar y } \\
\text { muñeca }\end{array}$ & De poder & Desvio & \multicolumn{2}{|c|}{0 Pie } & Derribos & Toma de cintura & $\begin{array}{l}\text { Subaxilar y } \\
\text { muñeca }\end{array}$ & 0 \\
\hline 270 & 19 & & & & & & \multicolumn{2}{|c|}{0} & Proyecciones & Suplex & Ninguno & 4 \\
\hline 321 & 51 & $\begin{array}{l}\text { Contra } \\
\text { ataque }\end{array}$ & Controles & Subaxilar doble & De poder & Movimiento & \multicolumn{2}{|c|}{2 Pie } & Derribos & Toma de cintura & & 0 \\
\hline 333 & 12 & Ofensiva & Proyecciones & Suplex & De poder & $\begin{array}{l}\text { Ataque } \\
\text { Directo }\end{array}$ & \multicolumn{2}{|c|}{2 Piso } & Controles & Rotacional & & 0 \\
\hline segundos & minutos & & & & \multirow[t]{2}{*}{3} & 3 & 4 total ptos & 6 & & & & total ptos \\
\hline 333 & 5,6 & & & & & & 10 & 2 & & & & 8 \\
\hline
\end{tabular}

Figura 2. Instrumento posterior al proceso de validación de contenidos (eliminación de la orientación, calidad de las acciones, ubicación y riesgo promedio). Fuente: elaboración propia

Se observa el mismo combate, pero resumiendo solamente los aspectos que fueron aceptados tras el proceso de validación de contenidos. 
Tabla 5.

Tabla de Aiken para establecer puntajes de corte basado en el número de expertos y la escala de calificación utilizada.

TABLE 1

Right-Tail Probabilities (p) for Selected Values of the Validity Coefficient (V)

\begin{tabular}{|c|c|c|c|c|c|c|c|c|c|c|c|c|}
\hline \multirow{3}{*}{$\begin{array}{c}\text { No. of Items } \\
(m) \text { or } \\
\text { Raters }(n)\end{array}$} & \multicolumn{12}{|c|}{ Number of Rating Categories (c) } \\
\hline & \multicolumn{2}{|c|}{2} & \multicolumn{2}{|c|}{3} & \multicolumn{2}{|c|}{4} & \multicolumn{2}{|c|}{5} & \multicolumn{2}{|c|}{6} & \multicolumn{2}{|c|}{7} \\
\hline & V & $\mathrm{p}$ & $\mathrm{V}$ & $\mathrm{p}$ & V & $\mathrm{p}$ & V & $\mathrm{p}$ & V & $\mathrm{p}$ & V & p \\
\hline 2 & & & & & & & 1.00 & .040 & 1.00 & .028 & 1.00 & .020 \\
\hline 3 & & & & & & & 1.00 & .008 & 1.00 & .005 & 1.00 & .003 \\
\hline 3 & & & 1.00 & .037 & 1.00 & .016 & .92 & .032 & .87 & .046 & .89 & .029 \\
\hline 4 & & & & & 1.00 & .004 & .94 & .008 & .95 & .004 & .92 & .006 \\
\hline 4 & & & 1.00 & 012 & .92 & .020 & .88 & .024 & .85 & .027 & .83 & .029 \\
\hline 5 & & & 1.00 & .004 & .93 & .006 & .90 & .007 & .88 & .007 & .87 & .007 \\
\hline 5 & 1.00 & .031 & .90 & .025 & .87 & .021 & .80 & .040 & .80 & .032 & .77 & .047 \\
\hline 6 & & & .92 & .010 & .89 & .007 & .88 & .005 & .83 & .010 & .83 & .008 \\
\hline 6 & 1.00 & 016 & .83 & .038 & .78 & .050 & .79 & .029 & .77 & .036 & .75 & .041 \\
\hline 7 & & & .93 & .004 & .86 & .007 & .82 & .010 & .83 & .006 & .81 & .008 \\
\hline 7 & 1.00 & .008 & .86 & .016 & .76 & .045 & .75 & .041 & .74 & .038 & .74 & .036 \\
\hline 8 & 1.00 & .004 & .88 & .007 & .83 & .007 & .81 & .008 & .80 & .007 & .79 & .007 \\
\hline 8 & .88 & .035 & .81 & .024 & .75 & .040 & .75 & .030 & .72 & .039 & .71 & .047 \\
\hline 9 & 1.00 & .002 & .89 & .003 & .81 & .007 & .81 & .006 & .78 & .009 & .78 & .007 \\
\hline 9 & .89 & .020 & .78 & .032 & .74 & .036 & .72 & .038 & .71 & .039 & .70 & .040 \\
\hline 10 & 1.00 & .001 & .85 & .005 & .80 & .007 & .78 & .008 & .76 & .009 & .75 & .010 \\
\hline 10 & .90 & .001 & .75 & .040 & .73 & .032 & .70 & .047 & .70 & .039 & .68 & .048 \\
\hline 11 & .91 & .006 & .82 & .007 & .79 & .007 & .77 & .006 & .75 & .010 & .74 & .009 \\
\hline 11 & .82 & .033 & .73 & .048 & .73 & .029 & .70 & .035 & .69 & .038 & .68 & .041 \\
\hline 12 & .92 & .003 & .79 & .010 & .78 & .006 & .75 & .009 & .73 & .010 & .74 & .008 \\
\hline 12 & .83 & .019 & .75 & .025 & .69 & .046 & .69 & .041 & .68 & .038 & .67 & .049 \\
\hline 13 & .92 & .002 & .81 & .005 & .77 & .006 & .75 & .006 & .74 & .007 & .72 & .010 \\
\hline 13 & .77 & .046 & .73 & .030 & .69 & .041 & .67 & .048 & .68 & .037 & .67 & .041 \\
\hline 14 & .86 & .006 & .79 & .006 & .76 & .005 & .73 & .008 & .73 & .007 & .71 & .009 \\
\hline 14 & .79 & .029 & .71 & .035 & .69 & .036 & .68 & .036 & .66 & .050 & .66 & .047 \\
\hline 15 & .87 & .004 & .77 & .008 & .73 & .010 & .73 & .006 & .72 & .007 & .71 & .008 \\
\hline 15 & .80 & .018 & .70 & .040 & .69 & .032 & .67 & .041 & .65 & .048 & .66 & .041 \\
\hline 16 & .88 & .002 & .75 & .010 & .73 & .009 & .72 & .008 & .71 & .007 & .70 & .010 \\
\hline 16 & .75 & .038 & .69 & .046 & .67 & .047 & .66 & .046 & .65 & .046 & .65 & .046 \\
\hline 17 & .82 & .006 & .76 & .005 & .73 & .008 & .71 & 010 & .71 & .007 & .70 & .009 \\
\hline 17 & .76 & .025 & .71 & .026 & .67 & .041 & .66 & .036 & .65 & .044 & .65 & .039 \\
\hline 18 & .83 & .004 & .75 & .006 & .72 & .007 & .71 & .007 & .70 & .007 & .69 & .010 \\
\hline 18 & .72 & .048 & .69 & .030 & .67 & .036 & .65 & .040 & .64 & .042 & .64 & .044 \\
\hline 19 & .79 & .010 & .74 & .008 & .72 & .006 & .70 & .009 & .70 & .007 & .68 & .009 \\
\hline 19 & .74 & .032 & .68 & .033 & .65 & .050 & .64 & .044 & .64 & .040 & .63 & .048 \\
\hline 20 & .80 & .006 & .72 & .009 & .70 & .010 & .69 & .010 & .68 & .010 & .68 & .008 \\
\hline 20 & .75 & .021 & .68 & .037 & .65 & .044 & .64 & .048 & .64 & .038 & .63 & .041 \\
\hline 21 & .81 & .004 & .74 & .005 & .70 & .010 & .69 & .008 & .68 & .010 & .68 & .009 \\
\hline 21 & .71 & 039 & .67 & .041 & .65 & .039 & .64 & .038 & .63 & .048 & .63 & .045 \\
\hline 22 & .77 & .008 & .73 & .006 & .70 & .008 & .68 & .009 & .67 & .010 & .67 & .008 \\
\hline 22 & .73 & .026 & .66 & .044 & .65 & .035 & .64 & .041 & .63 & .046 & .62 & .049 \\
\hline 23 & .78 & .005 & .72 & .007 & .70 & .007 & .68 & .007 & .67 & .010 & .67 & .009 \\
\hline 23 & .70 & .047 & .65 & .048 & .64 & .046 & .63 & .045 & .63 & .044 & .62 & .043 \\
\hline 24 & .79 & .003 & .71 & .008 & .69 & .006 & .68 & .008 & .67 & .010 & .66 & .010 \\
\hline 24 & .71 & .032 & .67 & .030 & .64 & .041 & .64 & .035 & .62 & .041 & .62 & .046 \\
\hline 25 & .76 & .007 & .70 & .009 & .68 & .010 & .67 & .009 & .66 & .009 & .66 & .009 \\
\hline 25 & .72 & .022 & .66 & .033 & .64 & .037 & .63 & .038 & .62 & .039 & .61 & .049 \\
\hline
\end{tabular}

Fuente: Tomado de Aiken (1985)

Debido al número de evaluadores (8 participantes) y escala de valoración (1 a 5) se determinó el punto de corte $(0,75)$ que se muestra en la tabla. 